\title{
Benchmarking and Performance Tuning of Multimedia Servers *
}

\author{
Peter Triantafillou, Stavros Christodoulakis and Theodora Magoulioti
}

MUltimedia Systems Institute of Crete, MU.S.I.C.

Department of Electronics and Computer Engineering

Technical University of Crete, T.U.C.

Chania, Crete, Greece ${ }^{\star *}$

\begin{abstract}
In this paper we study the issues related to the development of a multimedia server benchmark. Multimedia benchmarks can be used to compare the performance of existing multimedia servers and to fine-tune the performance of servers under development. The modeling of multimedia servers includes the modeling of databases, multi-user workloads, user behavior, and the definition of the relevant performance metrics. The proposed benchmark has been implemented and has been used to fine-tune the performance of a multimedia server, called $K Y$ DONIA. We present the results of testing KYDONIA and representative instances of fine-tuning KYDONIA's performance.
\end{abstract}

\section{Introduction}

Benchmark techniques have gained significant popularity and global acceptance which is widely reflected in the daily market life. As computer technology and computer products evolve nowadays, more and more the consuming society looks for realistic performance benchmarks, so as to have the correct attitude about the computer market. However, benchmarking is a difficult process because the goal of evaluation is typically ill-defined: End-users, sometimes even designers, either don't know, or can't specify exactly what result they expect. Often, they don't specify the architectural variants to consider, and often the metrics and workload they expect users to use are ill-defined. Moreover, they rarely clarify which kind of model and evaluation method best suit the evaluation problem.

In this paper, we study issues of systematic performance evaluation of large scale multimedia information systems, including DAVIC-compliant systems ([9], $[20]$ ). Such systems are typically geographically distributed and are centered on powerful multimedia servers whose high performance depends on exploiting parallel I/O mechanisms. We study the typical functionalities and requirements of the computer applications demanding multimedia access, the impact of user behavior on system performance, as well as the impact of data properties. The component performance metrics that most completely express the user's attitude about the overall system's efficiency were then determined. The objective is to

* This work was done in the context of and supported by the ESPRIT Long Term Research Project HERMES (project number 9141).

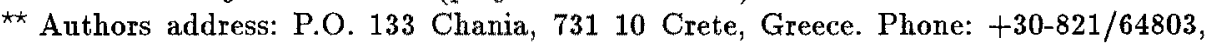
69737, 69738. FAX: +30-821/64846. E-mail address: \{peter,stavros,dora\}@ced.tuc.gr 
obtain benchmark results in order to evaluate the performance of the multimedia servers. To this end, it is important to mention that performance results depend significantly on the system architecture, which always must be revealed in full detail before characterizing the system performance ([18]).

The remainder of the paper is organized as follows. Section 2 describes the benchmark model. In Section 3 we show how we used the model to fine-tune the performance of the KYDONIA multimedia server ([5]). The corresponding results are described and analyzed in the same section. Section 4 concludes the paper and gives a short description of future work.

\section{The Benchmark Model}

We have developed and implemented a benchmark model in order to evaluate the performance of large scale multimedia information systems. The performance modeling of databases, multi-user workloads, and user behavior, as well as the definition of the performance metrics are the topics that must be addressed. The benchmark consists of three main parts, namely the Database Characterization, the Workload Characterization, and the Performance Metrics Definition. The parts are analyzed in the following sections.

\subsection{Database Characterization}

In general, database characterization involves the specification of the number of objects residing in the database, the characterization of database objects, and finally, the definition of the parameters of the scheme organization. In this paper we do not refer to scheme organization issues since it has been long studied by others ([3], [13]). However, we insist on object characterization analysis, which consists of two parts, namely object type information, and object replica information. Fig. 1 gives an overview of the database model.

Object Type Information We classify the objects into two types, namely atomic and composite.

An object of atomic type may be a delay-sensitive (ds), or a non-delaysensitive (nds) object. The $d s$ object type is the generic data type which can be used to describe time-dependent objects, such as video and audio sequences. Objects of this category consist of a sequence of media quanta (such as video frames and audio samples), which have to be presented in specified time intervals. A fundamental characteristic of the $d s$ objects is their large storage demands. Compression schemes are employed in order to limit them ([12], [15]). Different compression schemes correspond to different consumption data rate requirements. High data rates are typical of the $d s$ objects. Video and audio objects are currently the $d s$ instances studied here.

As $n d s$ we call the general data type which can be used to describe traditional textual and numeric data objects. Images are objects which also belong to this category. In general, they are characterized by simple attributes. For example, the attributes maintained within a text object is -likely- the text size and information concerning its structure and content. In addition, the information that image objects may hold includes size, resolution and compression schemes ([1], [17]). Images and text objects are representative classes of $n d s$ objects. 
Atomic objects can be combined in a fashion which usually requires synchronization. For example, a slide presentation must synchronize audio (music and commentary) with images. Thus, we define composite objects to consist of two or more atomic objects. Their basic feature is the number and type of atomic objects, as well as their interrelationships in time and space (e.g., atomic objects can start together, finish together, or start based on a lapse ([14], [19])).

Object Replica Information Replication may be desirable for performance and availability reasons, as well as for supporting different object qualities (e.g., multiple video resolutions). Consequently, we must first specify the number of copies for each database object, and second, we must characterize each copy based on its compression, size, and popularity information.

The $d s$ objects can be compressed by scalable compression algorithms, which create versions with different sizes ([16]). On the other hand, uncompressed objects (such as text) may exist in the database. We distinguish, therefore, two object categories, namely: uncompressed and compressed. Compressed objects may be characterized as having single, or multiple compressions. The JPEG and the MPEG standards are the compression schemes that typically are used for images and audio/video objects, respectively. We have also adopted multiple compression schemes which can also be used to implement the interactive VCR operations easily, because of the fact the multiple compression schemes can offer low, medium, high, and full data rate demands, simultaneously ([16]).

An object's type and compression information determine its size in bytes. A real database has objects of variable sizes. If, for instance, we consider the movie on demand service, then the subtitles, along with the compressed audio and video objects are representative database objects which are characterized by variable sizes. In general, we can consider that subtitles are small objects when compared with an audio object, which is a medium size object when compared to a video object. We define, therefore, three categories of objects based on their sizes: small objects, medium objects, and large objects. The lower and upper bounds of the ranges that contain small, medium and large objects, respectively, are given as parameters.

The frequency of access of real database objects varies significantly. Objects then are classified into two categories, namely hot and cold. The users access the objects of each class either uniformly, or according to an access pattern distribution. Also, the object's popularity varies with time.

\subsection{Workload Characterization}

In order to test the performance of multimedia information systems, we have to model actual multi-users workloads. The workloads denote representative tasks or service requests, which characterize the system's applications. To this end, we propose a workload model which consists of three tasks, namely functionality characterization, workload types characterization, and user population characterization. Fig. 2 overviews the workload model.

Functionality Characterization The design of a multimedia benchmark should allow the testing of all multimedia services that the system under test supports, such as video on demand ([9]). The services provide typical functionalities to 


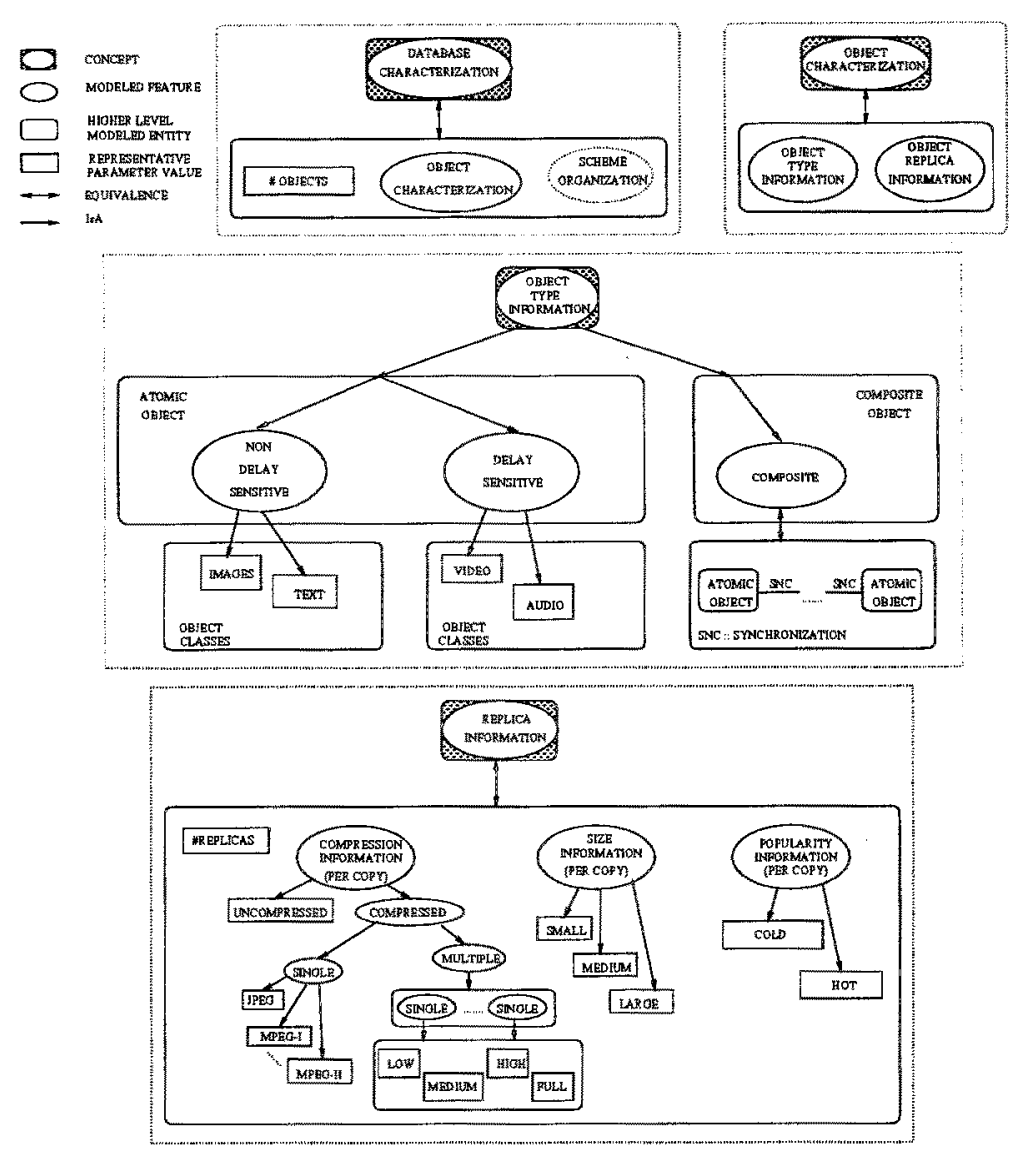

Fig. 1. An overview of the database model.

users. A benchmark needs to check whether typical service functionalities are provided. In our model we include the recommendation of DSM-CC ([10]), which has also been adopted by DAVIC ([9]), for defining the system's functionality. In this paper we consider the following functionalities:

1. Storing and retrieving various media types (e.g., text, audio, video, etc.).

2. Performing typical VCR operations (i.e., playback, FF, FB, stop, etc.).

Depending on whether user requests access atomic or composite objects, they are classified into two types, namely atomic and composite request types, respectively. The atomic requests are further classified into $n d s$ and $d s$ requests, depending on whether they access $n d s$, or $d s$ database objects, respectively.

Workload Types Modeled workloads consist of user sessions, each of which contains a number of requests. We distinguish two main types of multi-user workloads, namely simple and complex. Simple workloads are characterized by sessions that consist of only a single type of atomic requests (i.e., either $n d s$, or $d s$ requests). Complex workloads are classified into atomic and composite 
workloads. An atomic complex workload contains atomic requests of any type (i.e., both $n d s$ and $d s$ request type), while a composite and complex workload contains a set of composite requests.

User Characterization Users enter the system either with an exponential distribution arrival rate (in an open system), or according to the rules of a closed system. They create sessions which contain requests which typically follow a non-uniform access distribution. We have chosen to model the " $a / b$ rule", in which $a$ percent of accesses go to the most popular $b$ percent of the data, and the Zipf's Law distribution ([4], [24]).

To model the user's behavior, we must first decide which atomic/composite object (and if composite the constituent atomic objects and their synchronization requirements) will be accessed. Then for each request we must decide (i) whether it is a read/store, or VCR type request, (ii) on the object's type, (iii) whether the accessed object is compressed, or not, and (iv) on the object's size. Furthermore, we must define the number of sessions and the number of requests in each session which the workload contains. Finally, in addition to the arrival rate of users, in each session we define an arrival rate for its requests and (perhaps) a user's think time, in between the requests of a given session.

\subsection{Performance Metrics}

The system performance evaluation is based on measurements of numerous aspects of efficiency, which mainly depend on service specific features. The particular metrics are now presented.

Response Time and Startup Latency An aspect of response time can be defined as the cumulative time elapsed from submission of a request until reception of the whole answer. Representative requests involve the retrieval and/or the processing of traditional data. The usefulness of this definition depends on data length, since it is more suitable for the relatively smaller and $n d s$ objects. Indeed, considering requests that involve relatively larger objects, or $d s$ requests, we define response time as the time elapsed from submission of a request until the reception of a partial reply that can temporarily satisfy the user. For instance, a user who submits a playback video request realizes the server's response upon reception of enough video frames to begin its playback; not upon completion of the whole video playback. This is the start up latency metric defined, as the delay a user experiences from submission of a request until reception of the first units of data. Finally, it is important to measure the delay a user experiences from submission of a VCR request until system reaction to the user demands. Thus we define one more significant metric, called reaction time.

Throughput The throughput of a system is defined as the number of requests that are submitted and completed during a time unit. For example, concerning the movie on demand service, we can monitor the number of movies submitted and completed per hour $(m p h)$. The throughput typically expresses the maximum number of concurrent users that can be supported by the system. Various measurements of throughput can be reported depending on the response time restrictions enforced, the intra-/inter-object synchronization requirements enforced, the resolution requirements enforced, and at last, the system's price. 


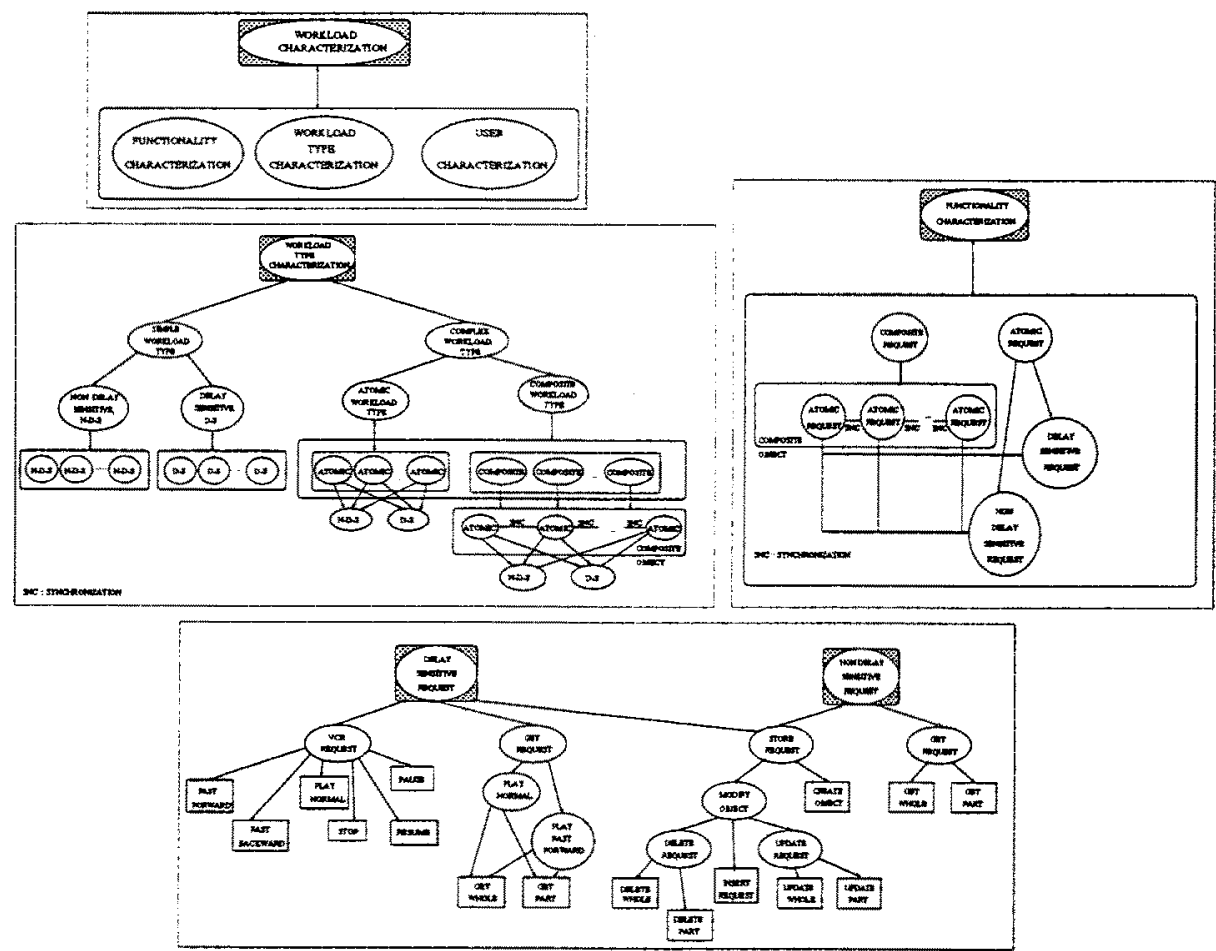

Fig. 2. An overview of the workload model. Functionality Characterization: Modeling of a single request. Workload Type Characterization: Modeling the resulting workload.

Throughput under Restrictions Apart from pure throughput reporting, several hybrid metrics can be defined. If, for example, we consider the response time metric, we can compute the percentage of requests that are actually serviced under user response time or startup latency constraints. A two-second delay was generally considered to be appropriate for "simple" commands, which are defined as those the user believes they can be done without any substantial processing and for which the user is not willing to wait. An example for a "simple" command is deleting an object. VCR requests are characterized by different user response time constraints, etc. User synchronization and presentation resolution constraints can also be considered.

Price/Throughput The cost of constructing and maintaining the system which can be either the hardware, software, or communication cost can be combined 
with the measured throughput that the priced configuration enables. The combination of throughput and associated price produces a new metric which is worth reporting.

Quality of Service A significant indicator of the system's efficiency is the Quality of Service, $Q_{0} S$, experienced by the user. The QoS is typically expressed in terms of the intra-object synchronization requirements, the inter-object synchronization requirements, and at last, the object presentation requirements. The $Q O S$ can be evaluated according to the function $Q \circ S=\mathcal{F}(\mathcal{H}, \mathcal{S}, \mathcal{R})$ where,

$\mathcal{H}$ : Delay-sensitive objects exhibit intra-object synchronization requirements. A failure of the system to meet the synchronization demands is perceived as disruptions and delays termed hiccups, or jitter. Hiccups are usually caused by delayed video frames, or audio samples. Consequently, data units must arrive at a specific rate and, therefore, deadlines are associated with the retrieval of successive data units. A cumulative characterization, $\mathcal{H}$, of deviation from intra-object synchronization requirements can be established via monitoring and reporting the total number of missed deadlines.

$\mathcal{S}$ : The interrelations in time and space among atomic objects that constitute a composite object is a special characteristic contributing in QoS metric. If a system fails to achieve the inter-object synchronization demands, then this can be perceived by users, for example, as audio being ahead of video, or inversely video being ahead of audio, Consequently, deadlines are associated with the retrieval of qualifying data units, as previously identified. A cumulative characterization, $\mathcal{S}$, of deviation from inter-object synchronization requirements can be established via monitoring and reporting the total number of missed deadlines.

$\mathcal{R}$ : This factor refers to video and image presentations on user display devices. Users request screen dimensions $S D_{1} \times S D_{2}$ that can be evaluated as the product $S D_{1} * S D_{2}$. However, the system supports the resolutions $R_{1} \times R_{2}$ that can be evaluated as the product $R_{1} * R_{2}$. The greater the product $R_{1} * R_{2}$, the better the resolution that the users experience. The bigger the difference $S D_{1} * S D_{2}-R_{1} * R_{2}$, the worse the resolution that the users experience. The mean (averaged over distinct requests) percentage of difference experienced is the quantity $\mathcal{R}$.

\section{Performance Tuning of the KYDONIA Server}

We currently use the benchmark presented above to observe and fine-tune $K Y$ DONIA ([5]). KYDONIA is a multimedia information server designed and implemented in MUSIC/TUC with the aim to investigate design issues and develop technology for the multimedia information systems of the future. KYDONIA's main features are: storage management for multimedia objects, real-time pumps towards multiple clients over ATM, multimedia DBMS functionality, multimedia object modelling, text and video access methods and browsing techniques. KYDONIA serves as a testbed for the research and development of multimedia information systems that support distributed multimedia applications, and with respect to this, a series of benchmarking tests takes place in order to improve overall server performance. In the following we present some of the benchmarks carried out along with the results. 
Benchmarks For our performance tests, we assume

- an MPEG-2 compression scheme with a data rate of 1.5 megabits per second per video stream. Based on this data rate, object sizes range from 576 kilobytes for a 3-second video to 11.25 megabytes for a 60 -second video. For our experiments, we assume all objects are 150 -second videos.

- that the server stores enough one-copy videos to fill available space.

- $d s$ workloads. We also assume that all objects are requested in their entirety and played sequentially. In reality, some percentages of the time users will deviate from sequential viewing to perform VCR operations, such as pause, fast backward and fast forward. Due on space constraints, we ignore them in this paper.

- that accesses to videos in the server follow a uniform distribution.

All measurements have been performed on the KYDONIA server (avoiding issues such as network bandwidth and client caching). Typical video on demand servers maintain strict performance constraints to guarantee adequate presentation quality; the data rate demands delivery of a video block as often as every (block_size_in_bytes $\div$ data_rate_demands_in_bytes) secs. The peak number of streams that can be supported without hiccups in presentation are the results of our experiments.

Results We show the performance of KYDONIA when qualifying videos have been stored in $m(=1,2,3)$ Seagate $S T 51080 N$ of size $1 \mathrm{~GB}$ and nominal bandwidth $T R_{d i s k}$ equal to $5 \mathrm{MB} / \mathrm{s}$ each disks and the video data block size varies in values of $1,2,3,4,5$, and $8 \mathrm{MB}$. The disks connect to individual SCSI controllers of nominal bus bandwidth $10 \mathrm{MB} / \mathrm{s}$ each. The data has been placed on the disks according to the fine-grained striping technique. In fine-grained striping, the striping unit is typically small. As a consequence, if there are $m$ disks, then every retrieval involves all the $m$ disk heads, and the $m$ disks behave like a single disk with bandwidth $m * T R_{d i s k}$. The following table shows the results on the throughput measurements. We obviously expect that as the number of disks increases and the data block size increases, throughput also increases.

\begin{tabular}{|c||ccc|}
\hline $\begin{array}{c}\text { Data Block Size } \\
\text { (in } M B)\end{array}$ & \multicolumn{3}{c|}{ Throughput } \\
$m=1$ & $m=2$ & $m=3$ \\
\hline 1 & 14 & 17 & 15 \\
2 & 15 & 26 & 28 \\
3 & 17 & 28 & 34 \\
4 & 17 & 31 & 36 \\
5 & 18 & 31 & 40 \\
8 & 19 & 35 & 46 \\
\hline
\end{tabular}

Discussion KYDONIA supports a maximum of 19 smooth streams (i.e., without hiccups) when using a single disk, and a maximum of 35 and 46 smooth streams when using 2 and 3 disks, respectively. Since multiplying by two and three the number of the involved disks definitely increases by a factor of two and three the disk bandwidth, the throughput doesn't follow a related increase! This is because in fine-grained striping the size of the striping unit decreases as 
the number $m$ of the involved disks increases, and this finally results in effective disk bandwidth decrease per disk!

Furthermore, by examining the design of the server closely and paying attention in KYDONIA's scheduling mechanism, we found that the current scheduler presents some important limitations. In particular, KYDONIA has incorporated a scheduling algorithm which is based on deadlines in order to meet the associated deadlines of the delay-sensitive objects. The deadlines are computed by taking into accounts only the following two factors; the consumption rate and the video data block size. Factors such as the load of the system are not taken into account. In addition, although the basic idea of the algorithm is to order all requests for disk access according to their associated deadlines (in increasing order), the server also performs SCAN scheduling in order to reduce the cost of positioning seeks (it takes place at KYDONIA's disk access module). This is obviously against philosophy of a deadline-based scheduling algorithm. The above result in missed deadlines ${ }^{1}$. In additional, we have seen that if for example all requests have the same deadline, some of them miss their deadline because of the fact that service rounds have interleaved (i.e., the block $j+1$ of a stream is retrieved before the block $j$ of another stream). Efforts are currently underway to re-examine very closely the scheduling of delay-sensitive requests in the KYDONIA server. Particularly we will implement the concept of round scheduling using an efficient algorithm like SCAN to process requests within a round. Round-based scheduling is known for solving such problems.

\section{Conclusions and Future Work}

We have developed a multimedia benchmark which consists of three parts, namely: Database Characterization, Workload Characterization and Performance Metrics Definition. Synthetic databases and multi-user workloads are realistically approximated by the first two parts, while the third part defines the performance metrics.

The benchmark can be used not only to evaluate the performance of a system in comparison to other similar systems, but also to fine-tune the performance of a given system. With respect to this, the proposed benchmark was used to fine-tune the KYDONIA server.

With respect to future work, our objective is to use the benchmark model to further fine-tune KYDONIA. In particular, we plan to incorporate in KYDONIA our work on striping techniques ([6], [23]), which will greatly improve its performance, and new scheduling algorithms, which will avoid the inefficiences of EDF-like algorithms. We will also incorporate our results on optimal data placement on multi-zones disks, disk arrays, and tertiary storage libraries to fine-tune those parts of KYDONIA managing these devices ([8], [21], [22], [23]). Finally, we will incorporate batching techniques to improve the performance.

${ }^{1}$ This can be avoided if the display of each stream is delayed until the end of the current round instead of starting the display as soon as the first block arrives in memory (as in currently done in KYDONIA). 


\section{References}

1. Anastasiadis. M., et al.: State of the Art: Image Compression Schemes. MU.S.I.C. Tech Report (1995) (in greek)

2. Böckle, G., et al.: Structured Evaluation of Computer Systems. IEEE Computer (June 1996) 45-50

3. Cattel. R., Skeen J.: Object Operations Benchmark. ACM Transactions on Database Systems (March 1992)

4. Chervenak. A.L.: Tertiary Storage: An Evaluation of New Applications. Doctor of Philosophy, Dept of Computer Science, Univ. of California, Berkeley (1994)

5. Christodoulakis. S., et al.: The KYDONIA Multimedia Information Server. To appear in ECMAST' 97

6. Christodoulakis. S., Zioga. F.: Fundamentals of Striping and Placement of DelaySensitive Data on Disks. (submitted for publication)

7. Christodoulakis. S., Triantafillou. P.: Research and Development Issues for LargeScale Multimedia Information Systems. ACM Computing Surveys (December 1995)

8. Christodoulakis. S., Triantafillou. P., Zioga F.: Principles of Optimally Placing Data in Tertiary Storage Libraries. VLDB '97 (accepted to appear)

9. DAVIC 1.0 Specification (1995-1996)

10. ISO/IEC JTC1/SC29/WG11: Digital Storage Media Command \& Control. (1996)

11. Quinn, S., R., Sitaram, M.: State of the Art: Testing, Testing. Byte (1996) 97-101

12. Gall, D.L.: MPEG: A Video Compressed Standard for Multimedia Applications. Communications of the ACM (vol. 34) (no. 4) (April 1991)

13. Gray, J.: The Benchmark Handbook for Database and Transaction Processing Systems. $2^{\text {nd }}$ edition, edited by Jim Gray Digital Equipment Corporation, Morgan Kaufmann Publishers, Inc., California

14. ISO/IEC CD 13522-5: Information Technology-Coding of Multimedia and Hypermedia Information. Part 5: MHEG Subset for Base Level Implementation (1995)

15. ISO/IEC 13818-3: Information technology - Generic coding of moving pictures and associated audio information -. Part 3: Audio (1995)

16. Keeton. K., Katz, R.: Evaluating Video Layout Strategies for a High Performance Storage Server. ACM/Verlag Multimedia Systems(3) (1995) 43-52

17. Kontogiannis. P., et al.: State of the Art: Image Formats. MU.S.I.C. Tech. Report (1996) (in greek)

18. Magoulioti. T., et al.: Benchmarking and Performance Aspects in Multimedia Information Systems. MU.S.I.C./HERMES, Tech. Report (1996)

19. Steinmetz, R., Nahrstedt, K.: Multimedia: Computing, Communications and Applications. Innovative Technology Series (1995)

20. Triantafillou, P., Christodoulakis, S.: Digital Libraries: A Survey of Developments in Required Technology. MU.S.I.C./HERMES Tech. Report, no. 5 (October 1996)

21. Triantafillou, P., Christodoulakis, S., Georgiadis, C.: Optimal Data Placement on Disks: A Comprehensive Solution for Different Technologies. IEEE Transactions on Knowledge and Data Engineering (conditionally accepted)

22. Triantafillou, P., Papadakis, T.: On-Demand Data Elevation in a Hierarchical Multimedia Storage Server. VLDB '97 (accepted to appear)

23. Triantafillou, P., Faloutsos, C.: Optimal Striping for Parallel I/O in Modern Applications. Parallel Computing journal, Special issue on Parallel Data Servers and Applications (accepted to appear)

24. Zipf. G.: The psycho-biology of language: An introduction to dynamic philology. Boston: Houghton Mifflin, 1935, Cambridge, MA:MIT Press (1965) 